\title{
Pilot studies in family planning and reproductive health care
}

\author{
Edwin van Teijlingen, Vanora Hundley
}

\section{Introduction}

This research methods paper outlines the different types of pilot studies, the most common reasons for using them and some of the key issues that need to be considered when planning, conducting and reporting pilot studies. The term pilot study can refer to so-called feasibility studies, which are "small scale version[s], or trial run[s], done in preparation for the major study", ${ }_{1}$ or to pre-testing or 'trying out' of a research instrument. ${ }^{2,3}$ Thus we can distinguish between a trial of the process and the piloting of an instrument (e.g. a questionnaire), both of which may be conducted as part of the research design stage. One of the benefits of conducting a pilot study is to get advance warning about where the main study could fall short, where research protocols may not be followed, and whether methods or instruments are inappropriate or too complicated for participants. In addition, pilot studies can convince funding bodies that the larger study is worth funding. Box 1 highlights the fact that pilot studies can fulfil a number of different roles at the same time.

\section{Box 1: Reasons for conducting pilot studies}

- Developing and testing adequacy of research instruments

Assessing the feasibility of a (full-scale) study/survey

- Designing a research protocol

- Assessing whether the research protocol is realistic and workable

- Establishing whether sampling frame and technique are effective

- Assessing the likely success of proposed recruitment approaches

- Identifying logistical problems that might occur using proposed methods

- Estimating variability in outcomes to help determining sample size

- Establishing initial contact with potential research participants

- Collecting preliminary data

- Determining what resources (finance/staff) are needed for planned study/survey

- Assessing the proposed data analysis techniques to uncover potential problems

- Developing a research question and/or research plan

- Training a researcher in as many elements of the research process as possible

- Training students as part of education in research methods

- Convincing funding bodies that research team is competent and knowledgeable

- Convincing funding bodies that the main study is feasible and worth funding

- Convincing other stakeholders that the main study is worth supporting

Adapted from van Teijlingen and Hundley (2001). ${ }^{4}$

J Fam Plann Reprod Health Care 2005; 31(3): 219-221

(Accepted 28 March 2005)

Dugald Baird Centre and Department of Public Health, Medical School, University of Aberdeen, Aberdeen, UK Edwin van Teijlingen, MEd, PhD, Medical Sociologist/Reader in Public Health

Department of Nursing and Midwifery, University of Stirling, Stirling, UK

Vanora Hundley, MSc, PhD, Honorary Senior Lecturer

Correspondence to: Dr Edwin van Teijlingen, Department of Public Health and Dugald Baird Centre for Research on Women's Health, University of Aberdeen, Polwarth Building, Medical School, Foresterhill, Aberdeen AB25 2ZD, UK.

Tel: +44 1224 552495. Fax: +44 1224550529.

E-mail: van.teijlingen@abdn.ac.uk

\section{Mixed methods}

The first phase, often referred to as an 'exploratory study', 3 may contain a number of different techniques to gather information to understand the various aspects of the phenomena being studied. Quantitative and/or qualitative methods may be used and large-scale studies might employ more than one study before the main survey is conducted. This exploratory stage is separate from pre-testing and should lead to the design of the research instrument. For example, indepth interviews or focus groups may help to establish the issues to be addressed in a large-scale questionnaire survey.

Next the instrument itself is piloted, for example, in a questionnaire the wording, the order of the questions, and the range of possible answers would be tested. At this stage it is important to establish that the instructions for the whole questionnaire and those for the individual questions are clear and not open to misinterpretation. The average time taken to complete the research instrument can also be established. One mistake often made in pilot studies is to test the draft research instrument, make changes and then send it out without a second round of piloting of the revised version. ${ }^{3}$

A feasibility pilot could then be conducted to test the research process, for example, the different ways of distributing and collecting questionnaires, or to identify potential practical problems in following the research procedure. The largest (decennial) survey in the UK, the Census (the latest was held in April 2001), tested the methodological and other changes made to the 1991 Census questionnaire on more than 100000 households. It "provided essential information on public reaction to new questions and form style as well as assessing the success of collection and processing methods". ${ }^{5}$ In a Scottish study of maternity care, the pilot demonstrated that the method of distributing the questionnaires, from the hospital records department, would not be adhered to. ${ }^{6}$ Without consulting the research team, the person responsible for distribution decided that it was better to go through the community midwives, despite the fact that the hospital had themselves suggested the records department.

Pilot studies can also identify whether selection or response bias will be a problem in the target sample. Boise et $a l$. discovered in their pilot study of reproductive health counselling during pregnancy in the USA that their participants were more likely to be older, married and "at a higher stage of change for contraceptive use at baseline ...". A focus in the full study on unmarried and/or younger women could lead you to consider the way the study is perceived and to produce recruitment materials for different subgroups in your target population. Other problems such as poor recording and response rates can also be identified and precautionary procedures or safety nets devised. The steps used to pilot a questionnaire on a small group, as similar as possible to the target population, are listed in Box 2 .

Pilot studies can also uncover local politics or problems that may affect the research process. van Teijlingen and colleagues found that maternity services managers had different perceptions about changes in the Data Protection Act (1998) and how this affected the involvement of their clients in research. ${ }^{6}$ One voiced ethical concerns about the use of reminder letters due to a previous local incident, where parents of an ill baby had been sent a questionnaire which was felt to be inappropriate, and as a result was wary 
Box 2: Pilot study procedures to improve internal validity of a questionnaire

Administer the questionnaire to pilot subjects in exactly the same way as it will be administered in the main study

- Ask the subjects for feedback to identify ambiguities and difficult questions

- Record the time taken to complete the questionnaire and decide whether it is reasonable

- Discard all unnecessary, difficult or ambiguous questions

- Assess whether each question gives an adequate range of responses

- Establish that replies can be interpreted in terms of the information that is required

- Check that all questions are answered

Re-word or re-scale any questions that are not answered as expected - Shorten, revise and, if possible, pilot again

Source: Peat et al. $\left(2002 ; 123\right.$, Table 3.23). ${ }^{8}$

of changes to the UK Data Protection Act. Consequently, reminders were sent out via the manager in case there were any problems with the newborn baby.

Feasibility studies can also demonstrate whether there is a problem getting practitioners or health policymakers to adopt an intervention or to make changes in policy. This may be valuable in persuading funding bodies to invest in a fullscale study. Cheyne et al. conducted a feasibility study of a decision aid to assist midwives in deciding whether a woman is in early labour. In addition to piloting the tool for validity and reliability, the study set out to examine the acceptability of the tool and whether maternity units would be willing to participate in the main study. 9 The results persuaded the funding body that the study was worth conducting and a large cluster randomised controlled trial (RCT) commenced in April 2005. Woodward and Kelly (2004) conducted a mini RCT with 80 women as part of their pilot study of water birth versus land birth. ${ }^{10}$ They found that randomisation did not affect the characteristics of the women using the pool and, therefore, health care professionals could have confidence in the results obtained from a larger RCT.

\section{Problems of pilot studies}

Pilot studies can have limitations. The findings of a pilot study can lead researchers to form inaccurate predictions or assumptions; problems can also arise from contamination; and there can be funding-related problems. First, completing a pilot study does not guarantee the success of the full-scale survey. Although pilot results may offer some indication of the likely size of the response rate in the main survey, they lack statistical foundation as they are usually based on small numbers. Other problems may not become obvious until the larger scale study is conducted.

A further concern is that of contamination. This may arise where: (1) data from the pilot study are included in the main study or (2) pilot participants are included in the main study, but new data are collected from them.

Social scientists engaged in predominantly quantitative research are likely to argue that: "an essential feature of a pilot study is that the data are not used to test a hypothesis or included with data from the actual study when the results are reported". ${ }^{11}$ The concern is that problems with the research tool and modifications made in the light of the pilot study could result in flawed or inaccurate pilot data. However, where an established and validated tool is being used and the pilot is determining other methodological aspects such as recruitment rates, it could be argued that such data may be of value.

A common problem with including pilot study participants in the main study is that such participants have already been exposed to an intervention and, therefore, may respond differently from those without such experience. ${ }^{4}$ This may be positive, for example, participants become more adept at using a new tool or procedure, or negative, with participants showing a decline in following a protocol because it is no longer new. Indeed both changes in behaviour have been recognised and therefore a 'run in' period, where an intervention is introduced prior to a study, is often used. The concern about including participants from the pilot study arises because only those involved in the pilot, and not the whole intervention group, will have had the experience. However, in some cases it is just not possible to exclude these participants because to do so would result in too small a sample in the main study. This is particularly a problem where the samples are clusters, for example, schools, family planning clinics or maternity hospitals. In such cases one can conduct a sensitivity analysis (or subgroup analysis) to assess to what extent the process of piloting influences the size of the intervention effect.

\section{Pilot studies in qualitative research}

Contamination is less of a concern in qualitative research where some or all of the pilot data are often used as part of the main study. Qualitative data collection and analysis is often progressive; a subsequent interview in a series should be 'better' than the previous one as the interviewer may have gained insights from previous interviews that are used to improve interview schedules and/or specific questions. As a result, separate pilot studies are not necessary in qualitative research because of its flexible nature. ${ }^{12}$ For example, a qualitative interviewer conducting 20 focus group interviews with women on the waiting list for in vitro fertilisation treatment will listen to the recordings and read through the transcripts of the first few focus groups in order to improve the questions, the way of introducing the issues to the group and add new topics. Thus although there is no specific pilot study, analysis of (and reflection on) the earlier focus groups can help improve the later ones. However, some argue that piloting provides the qualitative researcher with a "clear definition of the focus of the study", which in turn helps her to concentrate data collection in depth on a narrow spectrum of projected analytical topics. ${ }^{13}$ Piloting of qualitative approaches can also be carried out if "the researcher lacks confidence or is a novice, particularly when using the interview technique". ${ }^{12}$ For example, students can practise by doing pilot interviews on their fellow students or family, and supervisors, listening to the recording or reading the transcripts, can give advice on how to improve their interview style. Therefore, it is often advisable to conduct a small number of pilot interviews. ${ }^{14}$

Problems may also arise where a pilot study requires a significant investment of resources, making it difficult for the study team to stop the research after an unsuccessful pilot. Researchers might be tempted to make considerable changes in the main study, rather than deciding that it is not possible with the available resources. In contrast, funding bodies may be reluctant to fund a further study if the pilot has been substantial as they may view the research as no longer original, especially if the results are published.

\section{Why are pilot studies not reported?}

Publication bias may occur because journals tend to accept only papers that have statistically significant results. ${ }^{15-17} \mathrm{~A}$ study exploring research on passive smoking found a difference of 2 years in the median time to publication between studies with significant and non-significant results. ${ }^{18}$ Papers reporting methodological issues, such as those identified during the pilot, are often less attractive to journal editors.

Selective publication of research results has long been recognised as a problem. It may lead to an overestimation 
of the effectiveness of interventions, exposing patients to useless or harmful treatments, while overestimation of adverse effects may mean that patients are denied effective forms of care. ${ }^{19}$ In the past, editors have recognised the dangers of publication bias with respect to clinical trials and have offered 'an amnesty for unpublished trials' in an attempt to overcome this obstacle. ${ }^{20}$ However, it is equally important to ensure that lessons learned regarding research methods are shared, otherwise research participants may be subjected to poorly developed tools and money may be wasted because methods of recruitment failed. Consistent selection of primary research papers over research methods, theoretical thinking or secondary analysis papers can lead to researchers re-inventing the wheel and not having had the opportunity to learn from other people's experiences.

This leads us to the question of where such studies should be published. It might be tempting to think that pilot studies should only be reported in methodological journals, websites or databases where researchers can access them and thus decide on the best method for their particular study. However, we feel strongly that such studies also have valuable messages for consumers of research, the practitioner. An understanding of the challenges and limitations of conducting research is important if the practitioner is to be a discerning consumer of the evidence..$^{21}$ Several health journals publish methods papers that could include papers on pilot studies, for example, the Journal of Epidemiology and Community Health includes a section entitled 'Theory and Methods',22 whilst the Internet-based Journal of Experimental and Clinical Assisted Reproduction includes a section called 'Methodology' ${ }^{23}$ It is not always possible to report details of the pilot study alongside the main study due to word limitations, however editors might consider having joint papers thus providing the POEMs (patient-orientated evidence that matters) ${ }^{24-26}$ and information on how that was derived.

\section{Conclusions}

Well-conducted pilot studies can teach us a lot; unfortunately, full reports of pilot studies are rare in the reproductive health research literature though some good examples do exist.6,7,10,27 However, most reports only justify the research methods or particular research tool used. Too often research papers only refer to one element of the pilot study, for example, to the 'pre-testing' of a questionnaire. ${ }^{28}$ Such papers may simply state: "the questionnaire was tested for validity and reliability". When pilot studies are mentioned in more detail, researchers regularly comment that they "had learned from the pilot study" and made necessary changes, without offering details about what exactly has been learned. The processes and outcomes from both successful and failed pilot studies might be very useful to others embarking on projects using similar methods or instruments. This is particularly important because pilot studies can be "time-consuming, frustrating, and fraught with unanticipated problems, but it is better to ... deal with them before investing a great deal of time, money, and effort in the full study". ${ }^{29}$ It has been suggested that the current research climate demands accountability from researchers, thus there is a need to ensure the best possible use of research results. ${ }^{30}$ We argue that researchers have an ethical obligation to make the best use of their research experience by reporting issues arising from all parts of a study, including the pilot phase.

Well-designed and well-conducted pilot studies can inform about the best research process and occasionally about likely outcomes. Investigators should be encouraged to report their pilot studies, and in particular to report in more detail the actual improvements made to the study design and the research process.
Statements on funding and competing interests

Funding. None identified.

Competing interests. None identified.

References

1 Polit DF, Beck CT, Hungler BP. Essentials of Nursing Research: Methods, Appraisal and Utilization (5th edn). Philadelphia, PA: Lippincott Williams \& Wilkins, 2001; 467

2 Baker TL. Doing Social Research (2nd edn). New York, NY: McGraw-Hill Inc., 1994; 182-183.

3 Corbetta P. Social Research: Theory, Methods and Techniques. London, UK: Sage, 2003; 148-149.

4 van Teijlingen E, Hundley V. The importance of pilot studies. In: Gilbert N (ed.), Social Research Update Issue 35. Guildford, UK University of Surrey, 2001. http://www.soc.surrey.ac.uk/sru/ SRU35.html [Accessed 21 March 2005].

5 Office for National Statistics, General Register Office for Scotland, Northern Ireland Statistical and Research Agency. 2001 Census Information Paper. London, UK: Government Statistical Services, $1999 ; 15$.

6 van Teijlingen E, Rennie AM, Hundley V, Graham W. The importance of conducting and reporting pilot studies: the example of the Scottish Births Survey, J Adv Nurs 2001; 34: 289-295.

7 Boise R, Petersen R, Curtis KM, Aalborg A, Yoshida CK, Cabral R, et al. Reproductive health counseling at pregnancy testing: a pilot study, Contraception 2003; 68: 377-383

8 Peat J, Mellis C, Williams K, Xuan W. Health Science Research: A Handbook of Quantitative Methods. London, UK: Sage, 2002; 123.

9 Cheyne H, Hundley V, Dowding D, Mollison J, Greer I. A feasibility study for a cluster randomised trial to investigate the use of a decision aid for the diagnosis of active labour in term pregnancy. The Early aid for the diagnosis of active labour in term pregnancy. The Early
Labour Study in Scotland. Final report for the Chief Scientist Office, Labour Study in Scotland. Final report for the Chief Scientist Office,
Scottish Executive Department of Health, Edinburgh, UK, 2004.

10 Woodward J, Kelly SM. A pilot study for a randomised controlled trial of waterbirth versus land birth. Br J Obstet Gynaecol 2004; 111 537-545.

11 Peat J, Mellis C. Health Science Research: A Handbook of Quantitative Methods. London, UK: Sage, 2002; 57.

12 Holloway I. Basic Concepts for Qualitative Research. Oxford, UK: Blackwell Science, 1997.

13 Frankland J, Bloor M. Some issues arising in the systematic analysis of focus group material. In: Barbour R, Kitzinger J (eds), Developing Focus Group Research: Politics, Theory and Practice. London, UK: Sage, 1999; 154.

14 Hundley V, van Teijlingen E. The role of pilot studies in research RCM Midwives J 2002; 5: 372-374

15 Mahoney M. Publication prejudices: an experimental study of confirmatory bias in the peer review system. Cognit Ther Res 1977; 1: $161-175$.

16 Chann S. The epidemiology of unpublished randomised controlled trials. Clin Res 1982; 30: 234A.

17 Dickersin K. The existence of publication bias and risk factors for its occurrence. JAMA 1990; 263: 1385-1389.

18 Misakian A, Bero L. Publication bias and research on passive smoking. JAMA 1998; 280: 250-253.

19 Oxman AD, Cook DJ, Guyatt GH. Users' guides to the medical literature: VI. How to use an overview. Evidence-Based Medicine Working Group. JAMA 1994; 272: 1367-1371.

20 Smith R, Roberts I. An amnesty for unpublished trials. BMJ 1997; 315: 622 .

21 Oxman AD, Sackett DL, Guyatt GH. Users' guides to the medical literature: I. How to get started. JAMA 1993; 270: 2093-2095.

22 Journal of Epidemiology and Community Health. http://jech.bmjjournals.com/ [Accessed 21 March 2005].

23 Journal of Experimental and Clinical Assisted Reproduction. $\mathrm{http} / / / \mathrm{www}$.jexpclinassistreprod.com/articles/browse.asp?sort=Metho dology [Accessed 21 March 2005].

24 Slawson DC, Shaughnessy AF, Bennett JH. Becoming a medical information master: feeling good about not knowing everything. J Fam Pract 1994; 38: 505-513.

25 Slawson DC, Shaughnessy AF. Obtaining useful information from expert based sources. BMJ 1997; 314: 947 .

26 POEMs website: http://www.infopoems.com/ [Accessed 21 March 2005].

27 Lindquist R. Don't forget the pilot work! Heart Lung 1991; 20: 91-92.

28 De Vaus DA. Surveys in Social Research (3rd edn). London, UK: UCL Press, 1993

29 Mason DJ, Zuercher SL. Pilot studies in clinical nursing research. $J N$ Y State Nurs Assoc 1995; 26: 11.

30 Crosswaite C, Curtice L. Disseminating research results - the challenge of bridging the gap between health research and health action. Health Promot Int 1994; 9: 289. 